\title{
Enantioselective synthesis of epi-(+)-Sch 642305: observation of an interesting diastereoselection during RCM
}

\author{
Goverdhan Mehta* and Harish M. Shinde \\ Department of Organic Chemistry, Indian Institute of Science, Bangalore 560 012, India
}

\begin{abstract}
In an approach towards the enantioselective total synthesis of the novel bioactive natural product Sch 642305, an unusual diastereoselection during the key RCM reaction, resulted in the synthesis of the 11-epi-isomer of Sch 642305.
\end{abstract}

As part of a screening program in search of bacterial DNA primase (DnaG) inhibitors as potential new antibiotics, scientists at Schering-Plough recently reported the isolation of a novel natural product Sch 6423051 from the fermentation broth of the fungus Penicillium verrcosum (culture ILF-16214). ${ }^{1}$ The inhibition of E. Coli bacterial DNA primase enzyme by 1 with an $\mathrm{EC}_{50}$ value of $70 \mu \mathrm{M}$, though modest, constitutes an important lead towards developing new antibacterials. ${ }^{1,2}$ More recently and quite intriguingly, the presence of $\mathbf{1}$ has also been encountered in the isolates of the fungus Septofusidium sp. by a group at Merck and shown to exhibit inhibition of HIV-1 Tat-dependent transactivation with an $\mathrm{IC}_{50}$ value of $1.00 \mu \mathrm{M} .^{3}$ The structure and absolute configuration of $\mathbf{1}$ was elucidated by X-ray diffraction studies ${ }^{1}$ on its $p$-bromobenzoate derivative. While the unusual framework of 1, composed of a 4-hydroxycyclohexenone moiety fused to a decalactone moiety and the presence of four stereogenic centres makes it a challenging synthetic objective, it is the unique and broad-ranging ${ }^{1,3}$ biological activity profile of Sch 642305, which evokes special appeal towards a synthetic foray.

We have very recently accomplished ${ }^{4}$ the first total synthesis of $(+)$-Sch 6423051 following our second generation approach in which $\mathrm{C} 8-\mathrm{C} 9$ double bond formation through a RCM reaction was the pivotal step to generate the decalactone moiety. On the other hand, in our first generation approach to $\mathbf{1}$, retrosynthetically de-

\footnotetext{
* Corresponding author. Tel.: +91 8022932850; fax: +91 8023600936; e-mail: gm@orgchem.iisc.ernet.in
}

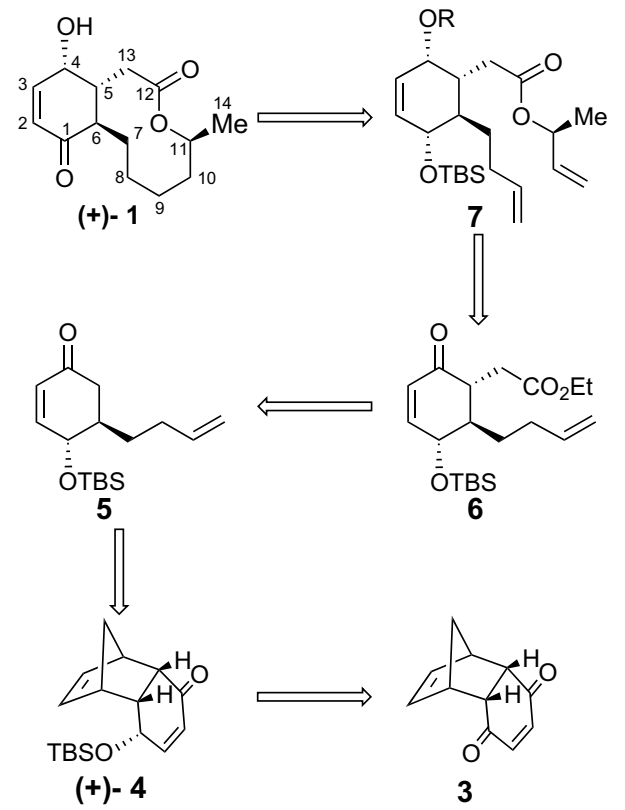

Scheme 1. Retrosynthetic analysis of (+)-Sch 642305.

picted in Scheme 1, we opted for the generation of the decalactone moiety on a preformed cyclohexenone platform through a RCM protocol involving the formation of the C9-C10 double bond. During the implementation of this approach (Scheme 1), we encountered an unexpected diastereoselection during the key RCM step and this culminated in the synthesis of 11-epi- $(+)-\mathrm{Sch}$ 6423052 rather than the natural product 1 . This interesting observation leading to the synthesis of $(+)-2$ forms the subject matter of this letter. 
According to the retrosynthetic theme displayed in Scheme 1, the previously described chiral synthon $(+)-$ $\mathbf{4}^{4,5}$ conveniently prepared from the readily available Diels-Alder adduct $\mathbf{3}$ of cyclopentadiene and $p$-benzoquinone, was to serve as our starting point. Stereocontrolled conjugate addition and retro-[4+2] reaction in $(+)-4$ was expected to deliver 5 in which sequential $\alpha$ alkylation to $\mathbf{6}$ and transesterification to 7 was expected to set up the key RCM reaction enroute to the natural product.

In actuality, $\mathrm{Cu}(\mathrm{I})$ mediated addition of butenylmagnesium bromide to the chiral synthon $(+)-4$ proceeded with good exo-face selectivity $(15: 1)$ to furnish $(+)-8{ }^{6}$ Thermal activation in $(+)-8$ disengaged the cyclopentadiene fragment through a retro-Diels-Alder reaction to deliver cyclohexenone (-)-5 (Scheme 2). Kinetically controlled $\alpha$-alkylation ${ }^{7}$ in $(-)-5$ proceeded with good stereoselection to furnish (-)-9 with the desired stereochemical orientation of the two side arms. ${ }^{6}$ Controlled Luche reduction ${ }^{8}$ in (-)-9 furnished a readily separable mixture of epimeric alcohols (-)-10 and (-)-11 (1.3:1), Scheme $2{ }^{6}$ The lack of stereoselectivity in the reduction of (-)-9 was not a major deterrent as the unwanted isomer (-)-10 could be readily oxidized to the enone (-)-9 and recycled. The stereochemistry of the required epimer (-)-11 was secured by its conversion to the $\gamma$-lactone 12 on exposure to titanium isopropoxide (Scheme 3). TBS-deprotection in $\mathbf{1 2}$ led to hydroxy-lactone 13 and further derivatization to the $p$-nitrobenzoate $\mathbf{1 4}$ furnished crystals suitable for X-ray structure determination $^{9}$ and an ORTEP diagram is displayed in Scheme 3.

The free hydroxyl group in (-)-11 was protected as the TBDPS derivative to give $(+)-\mathbf{1 5},{ }^{6}$ so that the two silicon protecting groups on the six-membered ring could be easily chemo-differentiated (Scheme 4). Transesterification $^{10}$ in $\mathbf{1 5}$ with racemic 3-buten-2-ol in the presence<smiles>C=CCCC1C(O)C=C[C@H]2OC(=O)C[C@H]2C1CCC=C</smiles>

$(-)-12$

$(-)-13$

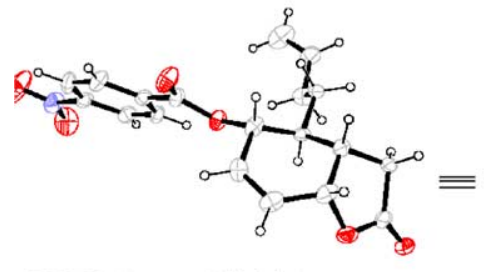

ORTEP disgram of (-)-14 drawn at $50 \%$ ellipsoidal probability

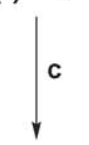<smiles>C=CCC1C(O)C=CC2OC(=O)CC21</smiles>

$(-)-14$

Scheme 3. Reagents and conditions: (a) $\mathrm{Ti}(\mathrm{O} i \mathrm{Pr})_{4}, \mathrm{EtOH}, 80^{\circ} \mathrm{C}, 2 \mathrm{~h}$, $90 \%$; (b) HF, pyridine, THF, rt, $12 \mathrm{~h}, 88 \%$; (c) $p$-nitrobenzoyl chloride, DIPEA, DMAP ( 0.1 equiv), rt, $7 \mathrm{~h}, 85 \%$.

of titanium isopropoxide proceeded smoothly and expectedly furnished a 1:1 diastereomeric mixture of esters 16a,b, the projected precursor for the key RCM reaction as per the retrosynthetic sequence shown in Scheme 1. Since, the diastereomers 16a,b were inseparable, they were as such subjected to the RCM reaction using the Grubbs' first generation catalyst 17.

The initial attempts to induce RCM in $\mathbf{1 6 a}, \mathbf{b}$ with Grubbs' catalyst ${ }^{11}$ were unsuccessful and attributing this failure to the intervention by unproductive chelation (see 18 and 19) by the evolving carbene species, as has been observed previously, ${ }^{12}$ we decided to explore $\mathbf{1 7}$ and $\mathrm{Ti}(\mathrm{OiPr})_{4}$ as a binary catalyst system. ${ }^{11,12 \mathrm{~b}}$

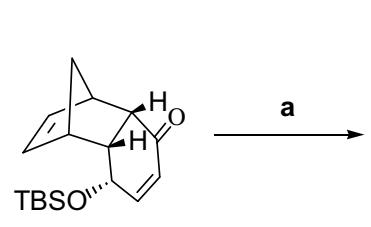

(+)- 4

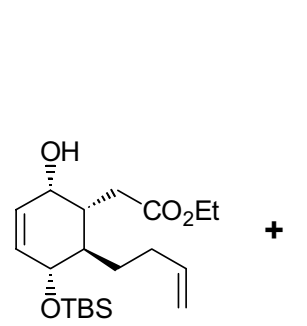

$(-)-11$
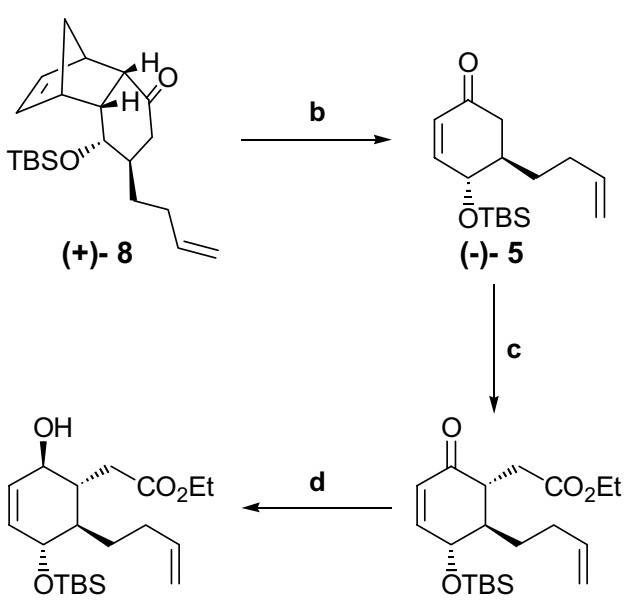

$(-)-10$
(-)- 9

Scheme 2. Reagents and conditions: (a) butenylmagnesium bromide, $\mathrm{CuI}, \mathrm{THF}, 85 \%(\alpha: \beta=1: 15)$; (b) $\mathrm{Ph}-\mathrm{O}-\mathrm{Ph}, 220{ }^{\circ} \mathrm{C}, 10 \mathrm{~min}, 80 \%$; (c) $\mathrm{LDA}$, $\mathrm{BrCH}_{2} \mathrm{CO}_{2} \mathrm{Et},-78^{\circ} \mathrm{C}, 60 \%$; (d) $\mathrm{NaBH}_{4}, \mathrm{CeCl}_{3}, \mathrm{MeOH},-50{ }^{\circ} \mathrm{C}, 80 \%(\mathbf{1 0 : 1 1}=1.3: 1)$; (e) PDC, $4 \AA$ A $\mathrm{MS}, \mathrm{DCM}, 1 \mathrm{~h}, 0{ }^{\circ} \mathrm{C}, 92 \%$. 


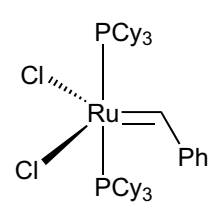

17

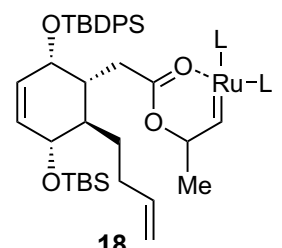

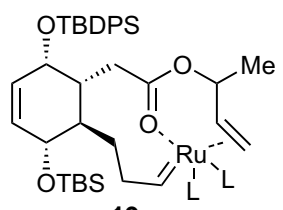

19

Indeed, exposure of 16a,b to Grubbs' catalyst $\mathbf{1 7}$ in the presence of $\mathrm{Ti}(\mathrm{OiPr})_{4}$ led to a single product $(+)-\mathbf{2 0}$ in modest yield and we were somewhat surprised to observe that this reaction did not proceed to completion and that only one of the diastereomers had undergone the RCM reaction although at this stage it was not clear as to which diastereomer had reacted. This finding was complemented by the observation that recovered 16a,b from this RCM reaction exhibited enrichment of the unreacted diastereomer. When we attempted to perform the RCM reaction under forcing conditions or for longer duration or with the Grubbs' second generation catalyst, ${ }^{13}$ the results were disappointing and mired with complications. However, unmindful of the exact C-11 methyl stereochemistry in $\mathbf{2 0}$ at this stage but drawing solace from the fact that we were dealing with a single diastereomer, we proceeded further. Regioselective hydrogenation in $(+)-\mathbf{2 0}$ was uneventful and led to $(+)-21$ (Scheme 4). Chemoselective deprotection ${ }^{14}$ in $(+)-21$ led to the allylic alcohol $(+)-22$, which was further oxidized with PDC to the corresponding enone and the TBDPS group was deprotected to furnish 2 and not the anticipated natural product $1 .^{6}$ The spectral characteristics of $\mathbf{2}$, though similar to that of $\mathbf{1}$, were not identical with that of the natural product. The formulation of 11-epi-Sch $642305(+)-2$ led us to surmise that during the key RCM reaction on $16 \mathbf{a}, \mathbf{b}$, only the $\alpha$ methyl diastereomer 16a reacted and this diastereoselection led exclusively to the formation of $(+)-\mathbf{2 0}$ (Scheme 4).

The sensitivity of the RCM reaction to substitution on the participating alkene moieties, steric environment in the vicinity of the alkenes and presence of ligating groups among other factors have been noted before. ${ }^{12}$ However, the profound effect of the stereochemistry of the vicinal substituent (methyl group) on the course of the RCM reaction of $\mathbf{1 6 a}, \mathbf{b}$ observed here is quite unusual. We do not have a ready explanation for this diastereoselection but steric interactions between the $\beta$-methyl group of $\mathbf{1 6 \mathbf { b }}$ and the $\beta$-oriented C6-alkene arm might be a causative factor that destabilizes the requisite alignment of the alkenes, thereby leaving the $\alpha$-methyl isomer 16a to undergo the RCM reaction to furnish 20.

In short, we have explored a RCM based enantioselective approach to the recently reported bioactive natural product Sch 642305. This effort has culminated in the synthesis of (+)-11-epi-Sch 642305 through an unusual diastereoselection during the RCM protocols.

\section{Acknowledgements}

H.M.S. thanks CSIR for the award of a research fellowship. X-ray data were collected at the CCD facility at IISc. We thank Mr. Atsuya Hirano and Mr. Tan Nakagawa, Amano Enzyme Co., Nagoya, Japan, for their generous gift of Lipase PS-D. This research was supported by the Chemical Biology Unit of JNCASR in Bangalore.

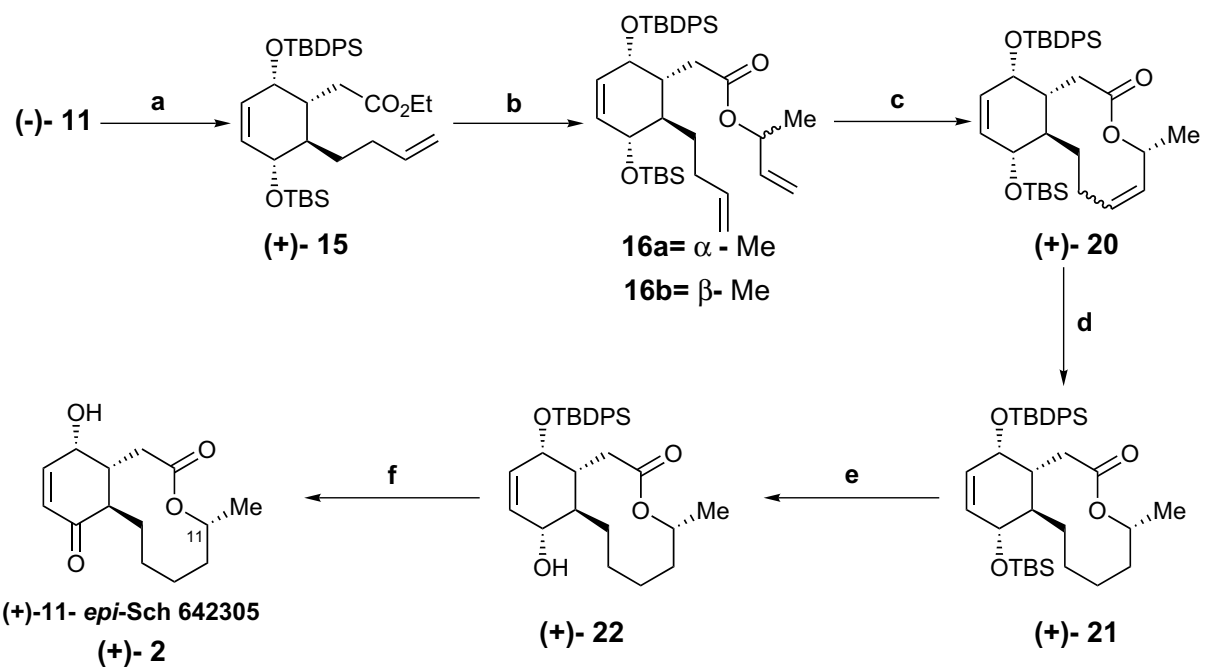

Scheme 4. Reagents and conditions: (a) TBDPSCl, imidazole, DMF, rt, 12 h, 92\%; (b) Ti(OiPr) 4 , 3-butene-2-ol, $90{ }^{\circ} \mathrm{C}, 10$ h, $83 \%$; (c) Grubbs' catalyst 17 (20 mol \%), Ti(OiPr $)_{4}$, benzene, $80{ }^{\circ} \mathrm{C}, 2 \mathrm{~h}, 30 \%$; (d) $\left(\mathrm{Ph}_{3} \mathrm{P}\right)_{3} \mathrm{RhCl}, \mathrm{H}_{2}(1 \mathrm{~atm}), 45 \mathrm{~h}, 77 \%$; (e) PPTS, EtOH, $81 \%, 12 \mathrm{~h}(95 \%$ based on recovered starting material); (f) (i) PDC, 4 A MS, DCM, 5 h, 92\%; (ii) TBAF, AcOH (1:1), THF, rt, 9 h, $91 \%$. 


\section{References and notes}

1. Chu, M.; Mierzwa, R.; Xu, L.; He, L.; Terracciano, J.; Patel, M.; Gullo, V.; Black, T.; Zhao, W.; Chan, T.; Mcphail, A. T. J. Nat. Prod. 2003, 66, 1527.

2. (a) Bouché, J.-P.; Zechel, K.; Kornberg, A. J. Biol. Chem. 1975, 250, 5995; (b) Arai, K.-I.; Kornberg, A. Proc. Natl. Acad. Sci. U.S.A. 1979, 76, 4308; (c) Richet, H.; Mohammed, J.; McDonald, L. C.; Jarvis, W. R. Emerg. Infect. Dis. 2001, 7, 319; (d) Grompe, M.; Versalovic, J.; Koeuth, T.; Lupski, J. R. J. Bacterial. 1991, 173, 1268; (e) Shrimankar, P.; Stordal, L.; Maurer, R. J. J. Bacterial. 1992, 174, 7689.

3. Jayasuriya, H.; Zink, D. L.; Polishook, J. D.; Bills, G. F.; Dombrowski, A. W.; Genilloud, O.; Pelacz, F. F.; Herranz, L.; Quamina, D.; Lingham, R. B.; Danzeizem, R.; Graham, P. L.; Tomassini, J. E.; Singh, S. B. Chem. Biodiversity 2005, 2, 112.

4. Mehta, G.; Shinde, H. M. Chem. Commun. 2005, 3703.

5. (a) Takano, S.; Higashi, Y.; Kamikubo, T.; Moriya, M.; Ogaswara, K. Synthesis 1993, 948; (b) Konno, H.; Ogaswara, K. Synthesis 1999, 1135; (c) Mehta, G.; Islam, K. Synlett 2000, 1473.

6. All new compounds were fully characterized on the basis of IR, ${ }^{1} \mathrm{H}$ NMR, ${ }^{13} \mathrm{C}$ NMR and mass data. Spectral data of selected compounds: $(+)-8[\alpha]_{\mathrm{D}}^{26}-31.4$ ( c 1.4, $\mathrm{CHCl}_{3}$ ); IR (neat) 2935, 2860, $1709 \mathrm{~cm}^{-1} ;{ }^{1} \mathrm{H} \mathrm{NMR}$ $\left(300 \mathrm{MHz}, \mathrm{CDCl}_{3}\right): \delta 6.16(\mathrm{dd}, J=5.4$ and $3.3 \mathrm{~Hz}, 1 \mathrm{H})$, $6.02(\mathrm{dd}, J=5.4$ and $3.3 \mathrm{~Hz}, 1 \mathrm{H}), 5.80-5.67(\mathrm{~m}, 1 \mathrm{H})$, $5.00-4.92(\mathrm{~m}, 2 \mathrm{H}), 3.90(\mathrm{~m}, 1 \mathrm{H}), 3.31$ (br s, $1 \mathrm{H}), 3.12$ (br s, $1 \mathrm{H}), 2.87-2.77(\mathrm{~m}, 2 \mathrm{H}), 2.36-1.72(\mathrm{~m}, 6 \mathrm{H}), 1.38-1.34(\mathrm{~m}$, 1H), 1.27-1.24 (m, 2H), $0.95(\mathrm{~s}, 9 \mathrm{H}), 0.14(\mathrm{~s}, 3 \mathrm{H}), 0.08(\mathrm{~s}$, $3 \mathrm{H}) ;{ }^{13} \mathrm{C}$ NMR $\left(75 \mathrm{MHz}, \mathrm{CDCl}_{3}\right): 212.61,138.29,136.98$, $135.35,114.59,73.47,51.98,49.46,47.20,45.92,44.80$, $43.58,36.55,31.39,30.50,25.95$ (3C), 18.12, -3.92, -4.87; HRMS calcd for $\mathrm{C}_{21} \mathrm{H}_{34} \mathrm{SiO}_{2} \quad(\mathrm{M}+\mathrm{Na}): 369.2226$ found: 369.2213 ; (-) $-9[\alpha]_{\mathrm{D}}^{25}-109.7$ ( c 0.72, $\left.\mathrm{CHCl}_{3}\right)$; IR (neat) $2930,2858,1735,1686 \mathrm{~cm}^{-1} ;{ }^{1} \mathrm{H}$ NMR $(300 \mathrm{MHz}$, $\left.\mathrm{CDCl}_{3}\right): \delta 6.78(\mathrm{dd}, J=10.2$ and $2.1 \mathrm{~Hz}, 1 \mathrm{H}), 5.95(\mathrm{dd}$, $J=10.2$ and $2.1 \mathrm{~Hz}, 1 \mathrm{H}), 5.85-5.70(\mathrm{~m}, 1 \mathrm{H}), 5.06-4.96$ $(\mathrm{m}, 2 \mathrm{H}), 4.41-4.37(\mathrm{~m}, 1 \mathrm{H}), 4.19-4.11(\mathrm{~m}, 2 \mathrm{H}), 2.78-2.54$ $(\mathrm{m}, 3 \mathrm{H}), 2.24-2.03(\mathrm{~m}, 3 \mathrm{H}), 1.79-1.76(\mathrm{~m}, 1 \mathrm{H}), 1.65-1.45$ $(\mathrm{m}, 1 \mathrm{H}), 1.27(\mathrm{t}, J=7.2 \mathrm{~Hz}, 3 \mathrm{H}), 0.92(\mathrm{~s}, 9 \mathrm{H}), 0.15(\mathrm{~s}$, $3 \mathrm{H}), 0.13(\mathrm{~s}, 3 \mathrm{H}) ;{ }^{13} \mathrm{C} \mathrm{NMR}\left(75 \mathrm{MHz}, \mathrm{CDCl}_{3}\right): 199.36$, 172.24, 152.36, 137.77, 127.57, 115.02, 69.92, 60.60, 46.26, 45.83, 32.17, 27.47, 25.70 (3C), 17.93, 14.15, 9.18, -4.14, -4.80; HRMS calcd for $\mathrm{C}_{20} \mathrm{H}_{34} \mathrm{SiO}_{4}(\mathrm{M}+\mathrm{Na}): 389.2124$ found: $389.2135 ;(-)-11[\alpha]_{\mathrm{D}}^{26}-27.5$ (c $\left.1.2, \mathrm{CHCl}_{3}\right)$; IR (neat) 3450, 2929, 2858, $1731.8 \mathrm{~cm}^{-1} ;{ }^{1} \mathrm{H} \quad \mathrm{NMR}$ $\left(300 \mathrm{MHz}, \mathrm{CDCl}_{3}\right): \delta 5.87-5.69(\mathrm{~m}, 3 \mathrm{H}), 5.06-4.94(\mathrm{~m}$, $2 \mathrm{H}), 4.19-4.10(\mathrm{~m}, 3 \mathrm{H}), 3.93(\mathrm{~d}, J=6.3 \mathrm{~Hz}, 1 \mathrm{H}), 2.67-$ $2.48(\mathrm{~m}, 2 \mathrm{H}), 2.23-2.03(\mathrm{~m}, 4 \mathrm{H}), 1.79-1.56(\mathrm{~m}, 2 \mathrm{H}), 1.49$ $1.37(\mathrm{~m}, 1 \mathrm{H}), 1.26(\mathrm{t}, J=7.2 \mathrm{~Hz}, 3 \mathrm{H}), 0.89(\mathrm{~s}, 9 \mathrm{H}), 0.08(\mathrm{~s}$, $3 \mathrm{H}), 0.07(\mathrm{~s}, 3 \mathrm{H}) ;{ }^{13} \mathrm{C} \mathrm{NMR}\left(75 \mathrm{MHz}, \mathrm{CDCl}_{3}\right): 174.30$, $138.30,132.83,128.46,114.68,69.33,65.28,60.51,41.22$, 37.33, 33.90, 30.08, 28.07, 25.76 (3C), 17.94, 14.19, -4.17, -4.79; HRMS calcd for $\mathrm{C}_{20} \mathrm{H}_{36} \mathrm{SiO}_{4}(\mathrm{M}+\mathrm{Na})$ : 391.2281 found: 391.2281; (+)-20 $[\alpha]_{\mathrm{D}}^{23}+87.5$ (c $\left.0.4, \mathrm{CHCl}_{3}\right)$; IR (neat) 2929, 2856, $1724 \mathrm{~cm}^{-1} ;{ }^{1} \mathrm{H}$ NMR $(300 \mathrm{MHz}$, $\left.\mathrm{CDCl}_{3}\right): \delta$ 7.72-7.66 (m, 4H), 7.47-7.34 (m, 6H), 5.74 (br s, $2 \mathrm{H}), 5.56(\mathrm{~d}, J=9.9 \mathrm{~Hz}, 1 \mathrm{H}), 5.40(\mathrm{dd}, J=10.2$ and $3.0 \mathrm{~Hz}, 1 \mathrm{H}), 5.34-5.30(\mathrm{~m}, 1 \mathrm{H}), 4.10(\mathrm{~s}, 1 \mathrm{H}), 3.85(\mathrm{br} \mathrm{s}$, $1 \mathrm{H}), 2.59-2.51(\mathrm{~m}, 1 \mathrm{H}), 2.40-1.58(\mathrm{~m}, 7 \mathrm{H}), 1.39(\mathrm{~d}$, $J=6.3 \mathrm{~Hz}, 3 \mathrm{H}), 1.04(\mathrm{~s}, 9 \mathrm{H}), 0.94(\mathrm{~s}, 9 \mathrm{H}), 0.08(\mathrm{~s}, 6 \mathrm{H})$; ${ }^{13} \mathrm{C}$ NMR $\left(75 \mathrm{MHz}, \mathrm{CDCl}_{3}\right): 175.22,136.14,135.91$, $134.38,134.20,133.36,129.94,129.85,129.46,127.76$,
127.27, 67.83, 41.36, 27.01 (3C), 25.82 (3C), 20.91, 19.56, $18.04,-3.83,-4.54$; HRMS calcd for $\mathrm{C}_{36} \mathrm{H}_{52} \mathrm{Si}_{2} \mathrm{O}_{4}(\mathrm{M}+$ $\mathrm{Na})$ : 627.3302 found: $627.3315 ; \quad(+)-22[\alpha]_{\mathrm{D}}^{23}+150.0$ (c $0.22, \mathrm{CHCl}_{3}$ ); IR (neat) $3449,2930,2855,1726 \mathrm{~cm}^{-1}$; ${ }^{1} \mathrm{H}$ NMR $\left(300 \mathrm{MHz}, \mathrm{CDCl}_{3}\right): \delta 7.66(\mathrm{t}, J=7.5 \mathrm{~Hz}, 4 \mathrm{H})$, $7.45-7.34(\mathrm{~m}, 6 \mathrm{H}), 5.62(\mathrm{~d}, J=9.6 \mathrm{~Hz}, 1 \mathrm{H}), 5.40(\mathrm{dd}$, $J=9.6$ and $4.8 \mathrm{~Hz}, 1 \mathrm{H}), 5.05-4.95(\mathrm{~m}, 1 \mathrm{H}), 4.07(\mathrm{t}$, $J=3.6 \mathrm{~Hz}, 1 \mathrm{H}), 3.91-3.86(\mathrm{~m}, 1 \mathrm{H}), 2.63-2.56(\mathrm{~m}, 1 \mathrm{H})$, $2.12-2.05(\mathrm{~m}, 3 \mathrm{H}), 1.97-1.79(\mathrm{~m}, 2 \mathrm{H}), 1.67-1.45(\mathrm{~m}, 7 \mathrm{H})$, $1.27(\mathrm{~d}, J=6.6 \mathrm{~Hz}, 3 \mathrm{H}), 1.04(\mathrm{~s}, 9 \mathrm{H}) ;{ }^{13} \mathrm{C}$ NMR (75 MHz, $\left.\mathrm{CDCl}_{3}\right): 175.44,136.19,135.89,134.39,133.72$, $133.38,129.89,129.60,129.38,127.78,127.31,72.08$, $71.20,69.59,41.86,39.24,31.57,27.08$ (3C), 24.79, 22.87, 19.56; HRMS calcd for $\mathrm{C}_{30} \mathrm{H}_{40} \mathrm{SiO}_{4}(\mathrm{M}+\mathrm{Na})$ : 515.2594 found: 515.2599; (+)-2 $[\alpha]_{\mathrm{D}}^{24}+110.0$ (c 0.4 , $\mathrm{CH}_{3} \mathrm{OH}$ ); IR (neat) $3437,2924,2853,1724,1681 \mathrm{~cm}^{-1}$; ${ }^{1} \mathrm{H}$ NMR $\left(300 \mathrm{MHz}, \mathrm{CDCl}_{3}\right): 6.97(\mathrm{dd}, J=9.9$ and $6.0 \mathrm{~Hz}, 1 \mathrm{H}), 5.94(\mathrm{~d}, J=9.9 \mathrm{~Hz}, 1 \mathrm{H}), 5.00-4.92(\mathrm{~m}, 1 \mathrm{H})$, $4.22(\mathrm{dd}, J=6.0$ and $3.3 \mathrm{~Hz}, 1 \mathrm{H}), 2.81(\mathrm{dt}, J=12.3$ and $3.9 \mathrm{~Hz}, 1 \mathrm{H}), 2.59-2.37(\mathrm{~m}, 3 \mathrm{H}), 2.10-1.99(\mathrm{~m}, 1 \mathrm{H}), 1.96$ $1.77(\mathrm{~m}, 2 \mathrm{H}), 1.72-1.41(\mathrm{~m}, 4 \mathrm{H}), 1.28(\mathrm{~d}, J=6.0 \mathrm{~Hz}, 3 \mathrm{H})$, $1.05(\mathrm{~m}, 1 \mathrm{H}) ;{ }^{13} \mathrm{C}$ NMR $\left(75 \mathrm{MHz}, \mathrm{CDCl}_{3}\right): 202.54,176.42$, $148.49,130.26,74.41,68.79,47.78,42.30,40.03,32.04$, 25.76, 22.73, 22.25, 21.40; HRMS calcd for $\mathrm{C}_{14} \mathrm{H}_{20} \mathrm{O}_{4}$ $(\mathrm{M}+\mathrm{Na}): 275.1259$ found: 275.1265 .

7. Podraza, K. F.; Bassfield, R. L. J. Org. Chem. 1989, 54, 5919.

8. Gemal, A. L.; Luche, J. L. J. Am. Chem. Soc. 1981, 103, 5454.

9. Crystal data: X-ray data were collected at $293 \mathrm{~K}$ on a SMART CCD-BRUKER diffractometer with graphite monochromated $\operatorname{MoK} \alpha$ radiation $(\lambda=0.7107 \AA)$. The structure was solved by direct methods (SIR92). Refinement was by full-matrix least-squares procedures on $\mathrm{F}^{2}$ using SHELXL-97. The non-hydrogen atoms were refined anisotropically whereas the hydrogen atoms were placed in geometrically idealised positions and constrained to ride on their parent atoms. Compound (-)-14: $\mathrm{C}_{19} \mathrm{H}_{19} \mathrm{NO}_{6}$, $\mathrm{MW}=357$, colourless crystal, crystal system: orthorhombic, space group $P 2{ }_{1} 2_{1} 2_{1}$ : cell parameters: $a=4.8563(7)$ $\AA, \quad b=15.617(2) \AA, \quad c=23.622(4) \AA, \quad V=1791.50$ $\AA^{3}, \quad Z=4, \quad D_{\mathrm{c}}=1.32 \mathrm{~g} \mathrm{~cm}^{-3}, \quad F(000)=752, \quad \mu=0.10$ $\mathrm{mm}^{-1}$. Total number of $1 \mathrm{~s}$ parameters $=235, \quad R 1=$ 0.0835 for $2558 F_{\mathrm{O}}>4 \operatorname{sig}\left(F_{\mathrm{O}}\right)$ and 0.1066 for all 3159 data. $w R 2=0.1888, \mathrm{GOF}=1.155$, Restrained $\mathrm{GOF}=$ 1.155 for all data. Crystallographic data (without structure factors) have been deposited with the Cambridge Crystallographic Data Centre (CCDC 275655).

10. Seebach, D.; Hungerbühler, E.; Naef, R.; Schnurrenberger, P.; Weidmann, B.; Züger, M. Synthesis 1982, 138.

11. For recent reviews on RCM, see: (a) Grubbs, R. H.; Chang, S. Tetrahedron 1998, 54, 4413; (b) Blechert, S.; Schuster, M. Angew. Chem., Int. Ed. 1997, 36, 2036; (c) Fürstner, A. Angew. Chem., Int. Ed. 2000, 39, 3012; (d) Trnka, T. M.; Grubbs, R. H. Acc. Chem. Res. 2001, 34, 18; (e) Handbook of Metathesis; Grubbs, R. H., Ed.; WileyVCH: Weinheim, Germany, 2003; Vol. 2.

12. (a) Ghosh, A. K.; Cappiello, J.; Shin, D. Tetrahedron Lett. 1998, 39, 4651; (b) Fürstner, A.; Langemann, K. J. Am. Chem. Soc. 1997, 119, 9130; (c) Koskinen, A. M. P.; Nevalainen, M. Angew. Chem., Int. Ed. 2001, 40, 4060 .

13. Scholl, M.; Ding, S.; Lee, C. W.; Grubbs, R. H. Org. Lett. 1999, 1, 953.

14. Blair, I. A.; Saleh, S.; Prakash, C. Tetrahedron Lett. 1989, $30,19$. 